Article

\title{
Facile Fabrication of Recyclable, Superhydrophobic, and Oleophilic Sorbent from Waste Cigarette Filters for the Sequestration of Oil Pollutants from an Aqueous Environment
}

\author{
Augustine O. Ifelebuegu *, Egetadobobari E. Lale, Fredrick U. Mbanaso \\ and Stephen C. Theophilus \\ School of Energy, Construction and Environment, Coventry University, Coventry CV1 5FB, UK; \\ lalee@uni.coventry.ac.uk (E.E.L.); ab0312@coventry.ac.uk (F.U.M.); ab2038@coventry.ac.uk (S.C.T.) \\ * Correspondence: A.Ifelebuegu@coventry.ac.uk; Tel.: +44-247-765-7690
}

Received: 1 July 2018; Accepted: 21 August 2018; Published: 23 August 2018

\begin{abstract}
The oil industry is plagued with regular incidences of spills into the environment, causing environmental damage to flora and fauna, especially in marine environments where spills easily travel long distances from their sources. This study was carried out to investigate a simple two-step process for the conversion of waste cigarette filters into a superhydrophobic and oleophilic sorbent for application in oil/water separation and spill clean-up. Ultrasonically cleaned filters were surface modified by chemical vapour deposition using methyltrichlorosilane. The results show that the functionalised waste filters achieved superhydrophobic properties with a water contact angle of $154 \pm 3.5^{\circ}$, adsorbing 16 to 26 times their weights in various oils, which is a better oil sorption performance than those of commercially available non-woven polypropylene adsorbents. Also, the sorption capacity did not significantly deteriorate after 20 cycles of reuse, with up to $75 \%$ sorption capacity retained. The surface modified filters demonstrated excellent water repellency, oil sorption, and recyclability showing their potential application for full scale oil spill clean-up.
\end{abstract}

Keywords: cigarette filters; adsorption; oil/water separation; hydrophobicity; recyclability

\section{Introduction}

There have been several spills associated with the exploration, exploitation, and transportation of crude oil. Several small spills occur daily during the production and transportation of crude oil [1], with large-scale spills reported from time to time. In recent history, large-scale spills have occurred, such as the Deepwater Horizon drilling rig explosion in the Gulf of Mexico in 2010 which lasted for three months and remains a landmark oil spill. This incident renewed the interest on research into the development of cheaper and more effective remediation technologies to help mitigate the effects of possible future oil spills in aquatic environments [2,3]. Approaches used in the remediation of spills include natural, chemical, and mechanical remediation. Bioremediation, however, is the eco-friendliest means of clean-up; however, it requires more time, and as such is used as adjuncts to other remediation technologies. The effectiveness of bioremediation is also affected by temperature, oxygen present, and organic species $[4,5]$.

Among the other methods, the use of oil-absorbent materials is one of the most economical and effective approaches, because of the low operational costs, easy operation, and high efficiency [3,6,7]. To date, three types of oil-absorbent material have been frequently used to handle oil contamination. They include organic natural products, such as wood, poplar, cotton, kapok fibre, and keratinous materials [3,8]; inorganic mineral products, such as zeolite and inorganic meshes [9]; and synthetic 
organic products, such as polyacrylate, polystyrene, polypropylene, and polyurethane [10]. Nevertheless, traditional oil-absorbing materials have some disadvantages, such as low oil absorption capacity and low oil-saving rate [11]. Thus, it is important that oil-absorbing materials with high absorption capacity and a good oil-saving rate be fabricated in a green and facile manner to accommodate the growing demand for economical remediation methods.

Cigarette filters are among the most prevalent forms of litter, with an estimated 4.5 trillion produced annually around the world [12]. Tobacco production involves the use of a wide range of chemicals, from the processes used in growing it to the various chemicals involved in the manufacture of the filters, with the probability of these chemicals finding their way into the finished products. Additional dangerous chemicals are introduced to the filters during use in the form of smoke and particulate matter, which all contribute to make the waste filters dangerous to the environment [13-15]. Novotny et al. [12] found that arsenic, nicotine, polycyclic aromatic hydrocarbons, and heavy metals are released into the environment by littered "roadside waste" cigarette butts. They further stated that chemicals in cigarette filter leachate can be acutely toxic to aquatic organisms, as they found leachates from smoked cigarette tobacco, smoked cigarette filters, and unsmoked cigarette filters to be acutely toxic to the freshwater cladoceran (Daphnia magna). Cellulose (raw material for cigarette filters) is the most abundant polymer on earth, making it an inexhaustible source of raw material for creating environmentally friendly products. It is mainly produced by plants for use as their skeletal component. Cellulose acetate, on the other hand, is a derivative of cellulose, which is fabricated by the reaction of cellulose pulp with acetic acid, in the presence of sulfuric acid as the catalyst. This is then followed by a reaction with acetic anhydride, which is then spun as cellulose acetate [16]. Various studies have been attempted for the recycling of waste cigarette filters, which constitutes a huge environmental menace. Zhao et al. [17] applied the waste as a corrosion inhibitor, while Liu et al. [18] reported the application in oil spill clean-up. The purpose of this study was to develop a facile method for the recycling of waste cigarette filters as a raw material for the fabrication of superhydrophobic and oleophilic material for application in oil water separation.

\section{Materials and Methods}

\subsection{Materials}

The reagents used in the study, including acetone, dimethyl-sulfoxide, ethanol, Sudan red, methyl-trichlorosilane (MTCS), and methyl-trimethoxisilane (MTMS), were purchased from Sigma Aldrich (Gillingham United Kingdom). All reagents used were laboratory-grade products. The oils used in the adsorption tests include vegetable oil, diesel oil, and 5W-30 motor oil (obtained from Esso Filling Station, Coventry, UK) and vacuum pump (VP) oil. The cigarette filters were collected from within the university-designated smoking areas. The equipment used in the study included a portable shredder, scanning electron microscope (Zeiss Sigma500, Zeiss, Birkerød, Denmark), oven (Gallenkamp, Loughborough, UK), freezer (BIOBASE BDF-86V340, Jinan, China), and a Kruss CAM200 drop shape analyser (Hamburg, Germany).

\subsection{Fabrication of Adsorbent}

The fabrication process for the adsorbent started with the collection and cleaning of the filters before shredding. The shredded cigarette filters were soaked in ethanol under utrasonication for $1 \mathrm{~h}$ to remove impurities, and were subsequently hydrolysed in 1 molar sodium hydroxide solution for $10 \mathrm{~min}$ to enhance the roughness of the surfaces of the filters [18]. The filters were washed in distilled water until the supernatant was clear. They were then dried at $70{ }^{\circ} \mathrm{C}$ in an oven for $24 \mathrm{~h}$ before the surface modification. Due to the hydrophilic nature of the filters, the porous surfaces of the filters were then coated with methyl-trichlorosilane/methyl-trimethoxisilane by thermal vapour deposition method. The process involved putting $15 \mathrm{~mL}$ of the silylation solvents in $25 \mathrm{~mL}$ beakers, then transferring the beakers into a large glass container with a lid holding the sorbents. The glass 
containers now containing the $25 \mathrm{~mL}$ beakers with silylation solvents and sorbents were capped and put in an oven at $70{ }^{\circ} \mathrm{C}$ for $4 \mathrm{~h}$ to ensure complete coating of the sorbents, as graphically described in Figure 1. The functionalised sorbents were then allowed to cool and dry under atmospheric conditions for one day [4].

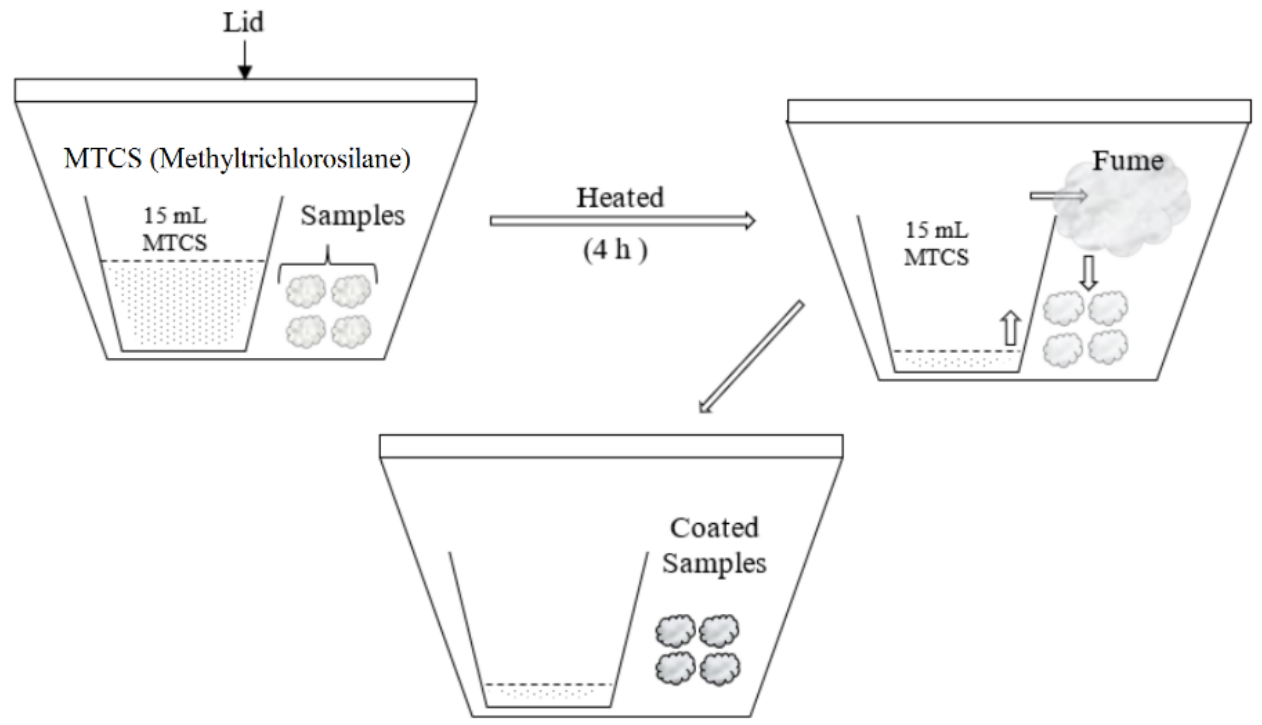

Figure 1. Schematic for the hydrophobic coating process.

\subsection{Characterisations of Adsorbents}

The changes in the morphology of the sorbents were observed before and after functionalisation by visual examination and with the aid of scanning electron microscopy. The porous surfaces were observed before and after functionalisation to ascertain the changes brought about by the silylation coating [19-21].

The hydrophobicity was determined, as have been previously described [22,23]. Water contact angles were measured using the sessile drop technique by making static measurements of the water contact angle on sorbent surfaces, with contact angle fitting performed according to the Young-Laplace fit $[7,24]$. Ten measurements were made for each sorbent at spatially different locations using HPLC-grade water $(0.4 \mu \mathrm{L})$, and the average water contact angles were reported.

\subsection{Batch Adsorption Tests}

Oil sorption characterization was carried out using a modified version of the method used by Feng et al. [4], which involved weighing the dry sorbent materials and then immersing them in $150 \mathrm{~mL}$ of the various oils for $2 \mathrm{~h}$ to ensure swelling equilibrium, after which they were lifted with a pair of tweezers, drained in the air for $30 \mathrm{~s}$, and weighed again. The oil absorption capacities of the filters were then calculated using $Q_{t}=\frac{m_{w}-m_{d}}{m_{d}}$ where $Q_{t}$ is the absorption capacity of the sorbent materials at time $t, m_{d(\mathrm{~g})}$ is the sorbent material weight before oil adsorption, and $m_{w(\mathrm{~g})}$ is the sorbent material weight after oil adsorption.

The recyclability of the sorbents was measured using the squeezing technique. The process involved carrying out several adsorption tests using the same sorbent material, squeezing into a beaker to recover the oil, and repeating the process. A total of twenty adsorption runs were carried out on the sorbents to test their recyclability [21,22,25]. 


\section{Results and Discussion}

\subsection{Surface Modification of the Adsorbents}

The adsorbent filters were functionalised using methyl-trimethoxisilane (MTMS) and methyltrichlorosilane (MTCS), which are both silylation reagents. The process involved heating a container that contained a beaker of the silylation reagents and the sorbents at $70{ }^{\circ} \mathrm{C}$ for $4 \mathrm{~h}$ for full coating of the sorbents, after which the sorbents were left under atmospheric conditions for one day. The MTCS was then absorbed to the surface, where the silane reacts with water to form siloxanes, creating nanoscopic roughness on the surface of the filter strands [26,27]. At the end of the process, the sorbents coated with MTCS showed excellent hydrophobic properties, as they all floated in water, and the roll-off angle of the functionalized samples were in all cases less than $2.2^{\circ}$. This was confirmed when a comparison was made between the un-functionalised and functionalised sorbents, as seen in Figure 2. All the un-functionalised sorbents sank to the bottom of the beakers, while the functionalised ones remained afloat. The filters functionalised with MTMS did not show the desired hydrophobicity. All subsequent tests were performed with MTCS surface-functionalised filters.

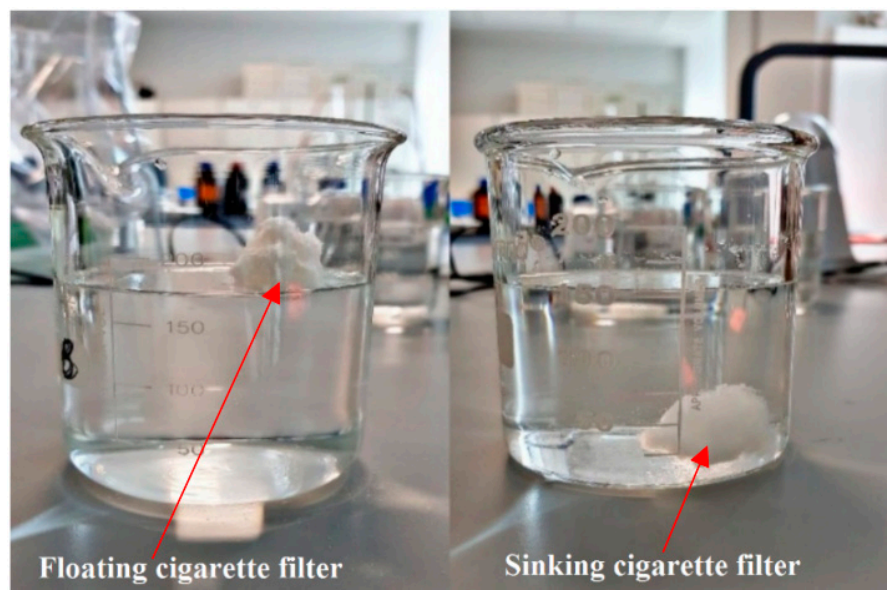

(a)

(b)

Figure 2. Hydrophobicity test using the floating method, between (a) functionalised cigarette filters and (b) un-functionalised cigarette filters.

The sorbents were dipped in beakers containing water and exhibited a silvery mirror-like appearance (Figure 3), which signifies that tiny air bubbles were trapped on the surface of the sorbents, thereby demonstrating hydrophobic behaviour as was also described by Ren et al. [22] and Han et al. [23].

\subsection{Surface Characterisation of Adsorbent Filters}

The surface morphology of the fabricated sorbent material was observed using a scanning electron microscope at an electron high tension (EHT) of $3 \mathrm{kV}$, a magnification of 992 times, and variable working distances between several runs at $10 \mu \mathrm{m}$ and $20 \mu \mathrm{m}$. The results show the surface morphologies of the sorbents with the un-functionalised variants (Figure 4a) exhibiting smooth surfaces on their individual strands. The functionalised variants (Figure $4 \mathrm{~b}$ ), however, showed the presence of the silylation coating, as seen by the grainy appearance on the surface of their individual strands. 


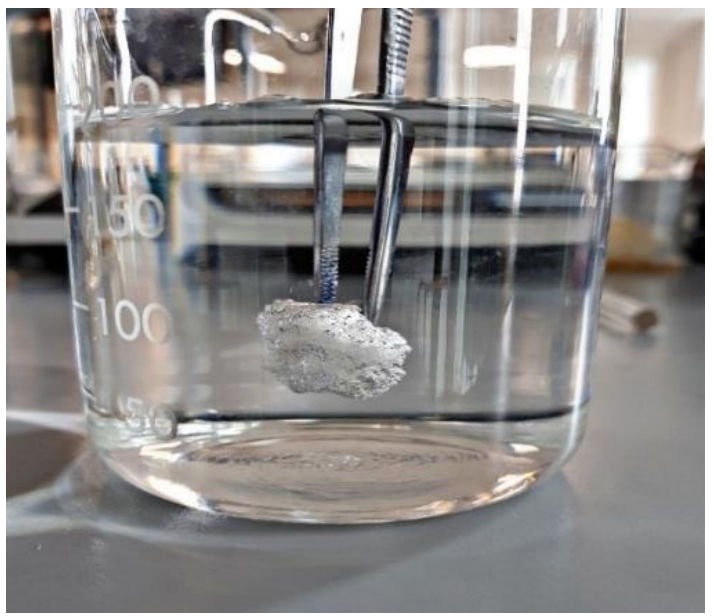

Figure 3. Hydrophobicity test showing air bubbles trapped on the surface of a functionalised cigarette filter.

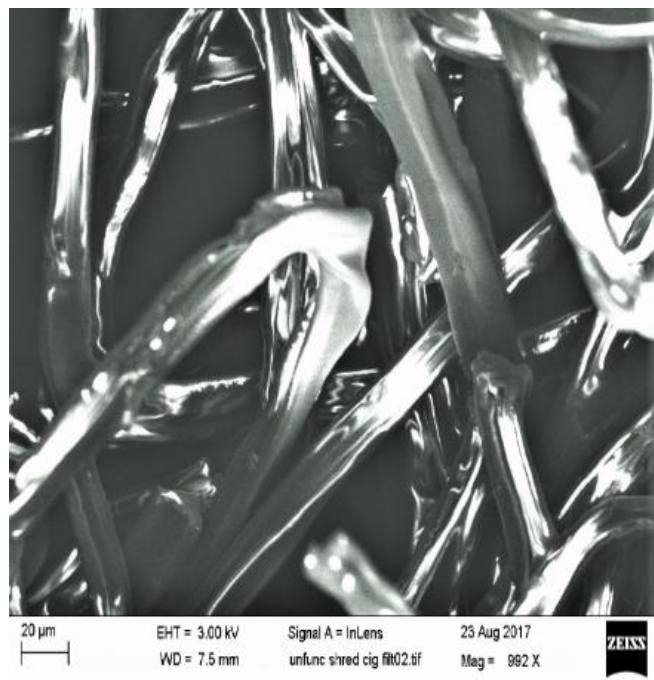

(a)

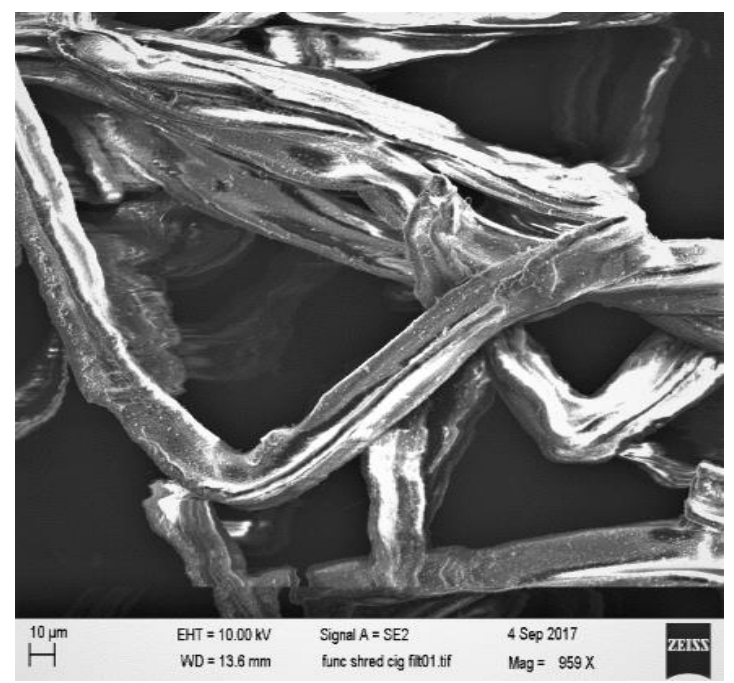

(b)

Figure 4. (a) Un-functionalised cigarette filter surface morphology; (b) functionalised cigarette filter surface morphology.

An ATR-FTIR (ThermoFischer Scientific, MA, USA) was carried out on the functionalised and un-functionalised filters. The results (Figure 5) confirm that the filters were made of cellulose acetate. The obvious difference in the curves is the emergency of a new peak at about $1200 \mathrm{~cm}^{-1}$, which is attributed to the symmetric and asymmetric stretching of the silicon bond, confirming the silanisation of the filters during the vapour condensation reaction.

Furthermore, to test for hydrophobicity, the water contact angles (CA) of the fabricated sorbent materials were tested using the Kruss CAM200. Ten measurements were made for each sorbent at spatially different locations, and the average was calculated. 


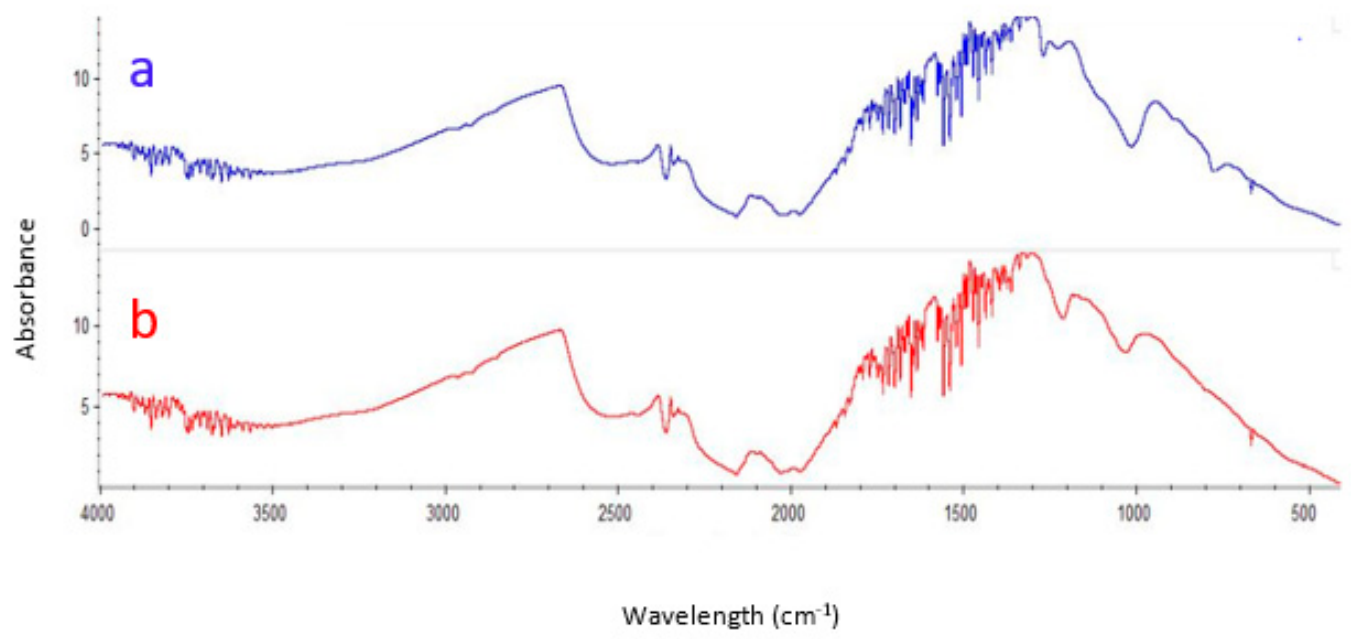

Figure 5. AT-Infra Red spectra of (a) functionalised cigarette filters and (b) unfunctionalized cigarette filters.

The analysis showed a maximum average water contact angle of $154 \pm 3.5^{\circ}$ and a roll-off angle of $2.2^{\circ}$ for the surface functionalized cigarette filters, indicating superhydrophobic properties, as can be seen in Figure 6, where adsorbent filter repelled water but adsorbed both diesel and vacuum pump oil. The CA values are comparable to those reported by other studies for carbon aerogel (132 $)$ [23], waste newspaper aerogel $\left(136^{\circ}\right)$ [24], silica cellulose aerogel $\left(138^{\circ}\right)$ [17], and octadecyltrichlorosilane modified cigarette filters $\left(158^{\circ}\right)$, cellulose aerogel $\left(150^{\circ}\right)$ [4] and MWCNTs-Silica aerogel $\left(154^{\circ}\right)$ [28]. The water contact angles for the un-functionalised cigarette filters was less than $10^{\circ}$.

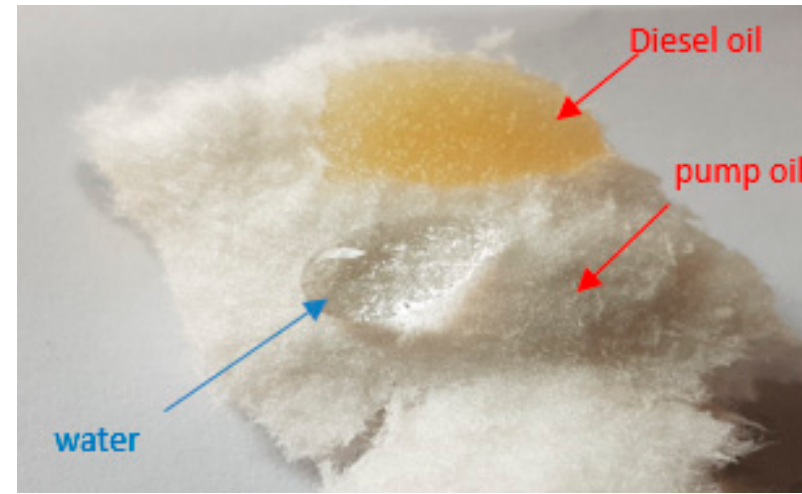

(a)

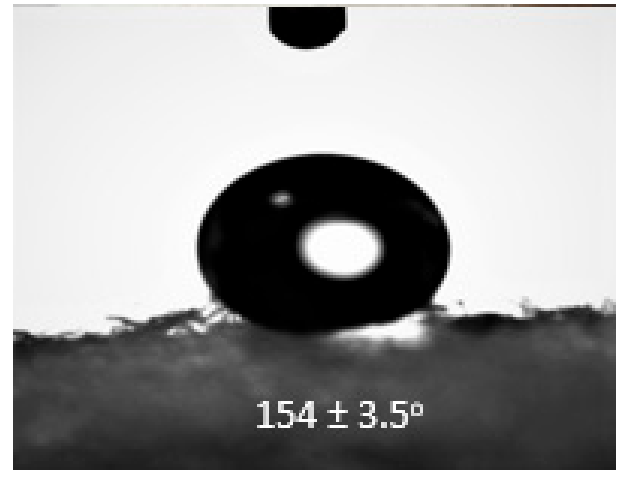

(b)

Figure 6. (a) Water/oil adsorption interaction and (b) the water contact angle for a functionalised cigarette filter.

To test the oleophilicity of the functionalised sorbents, a water-oil mixture was used. The method involved using a negligible quantity of Sudan red to colour motor oil, which was then added to a $250 \mathrm{~mL}$ beaker containing water [23]. The functionalised sorbents were then introduced to the mixture, where they demonstrated selective adsorption for oil. The sorbents adsorbed all the oil from the mixture within 1 min, leaving behind all the water, as seen in Figure 7. 


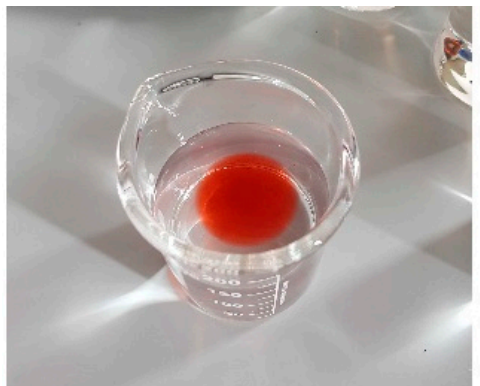

(a)

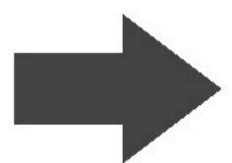

(b)

Figure 7. Selective adsorption schematic by sorbents. (a) Water and oil; (b) water oil and adsorbent filters.

\subsection{Oil Adsorption Capacities of Cigarette Filters}

The functionalised sorbents showed differential adsorption for the different oils used in the study. The order of the oil sorption capacity was vegetable oil $>$ vacuum pump oil $>$ motor oil $>$ diesel oil, which corresponds to the order of their densities, as shown in Figure 8. The results showed a superior performance of the surface-modified cigarette filters compared to commercial non-woven polypropylene sorbents, which were reported to have adsorption capacities of 9.1 to $10 \mathrm{~g} / \mathrm{g}$ for crude oil [29].

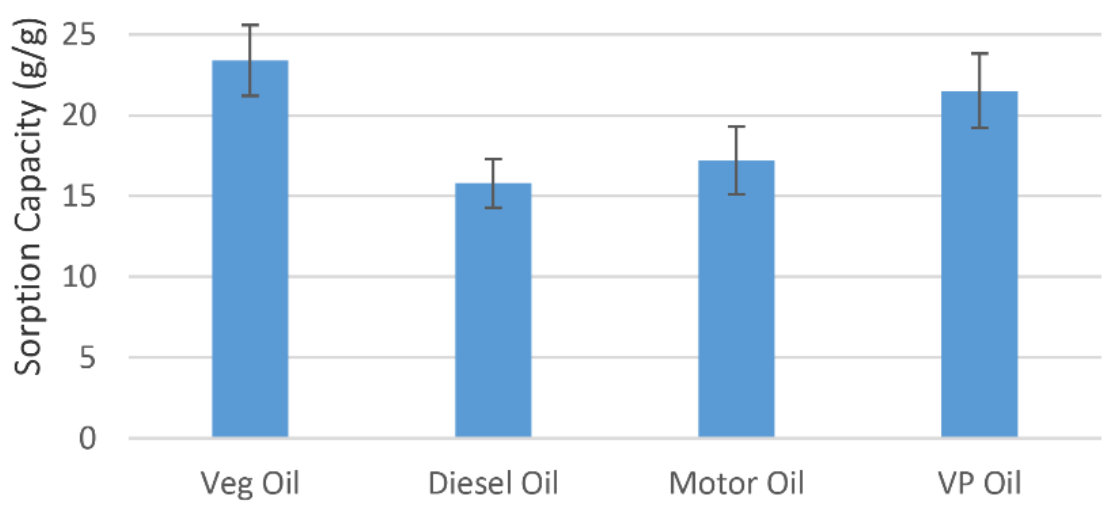

Figure 8. Sorption capacities of fabricated sorbents for various oils.

Table 1 presents the comparison of the sorption capacity of the waste cigarette butts with those of other oil adsorbents. As can be seen, they showed a better performance compared to commercial polypropylene, carbonised rice husk, and coconut coir, and compared favourably with graphene aerogel and carbon foam. The performance is seen to be less successful than that of cellulose aerogel. However, the filters used in this work have the advantage of easy fabrication (a simple two-step process), and can be made from readily available waste material. 
Table 1. Adsorption capacity of various fabricated sorbent materials.

\begin{tabular}{lccc}
\hline \multicolumn{1}{c}{ Adsorbent Material } & Oil Type & Adsorption Capacity (g/g) & References \\
\hline Polypropylene & Crude oil & $9.1-10$ & {$[29]$} \\
Superhydrophobic cotton & Crude oil & 52.6 & {$[30]$} \\
Graphene aerogels & Motor oil & 26 & {$[22]$} \\
Recycled cellulose aerogels & Motor oil & $18-20$ & {$[25]$} \\
Coconut coir fibre & Diesel oil & 6.53 & {$[31]$} \\
Recycled cellulose aerogels & Motor oil & $40-95$ & {$[4]$} \\
Cellulose Aerogel & Pump oil & 59.32 & {$[32]$} \\
Carbonised rice husk & Diesel oil & 5.5 & {$[33]$} \\
Carbon foam & Motor oil & 25 & {$[34]$} \\
\hline & Veg oil & 25.6 & \\
Cigarette filters & Diesel oil & 15.8 & This study \\
& Motor oil & 17.2 & \\
\hline
\end{tabular}

\subsection{Recyclability of Cigarette Filters and Shredded Cigarette Filters}

Sorbent recyclability (like buoyancy) and water repellency are important characteristics of a practical oil sorbent. The recyclability tests were carried out using the squeezing method, as the oil was easily recoverable by squeezing. The squeezing and reuse cycle was repeated 20 times using motor oil. It can be observed in Figure 9 that the sorption capacity of the filters decreased quickly within the first five repeat cycles, and then remained relatively stable for the next 15 cycles, retaining up to $75 \%$ of its oil sorption capacity after 20 cycles of reuse. The initial fast drop in adsorption capacity can be attributed to the recovery method. With the squeezing method, it is usually impossible to recover all the adsorbed oil.

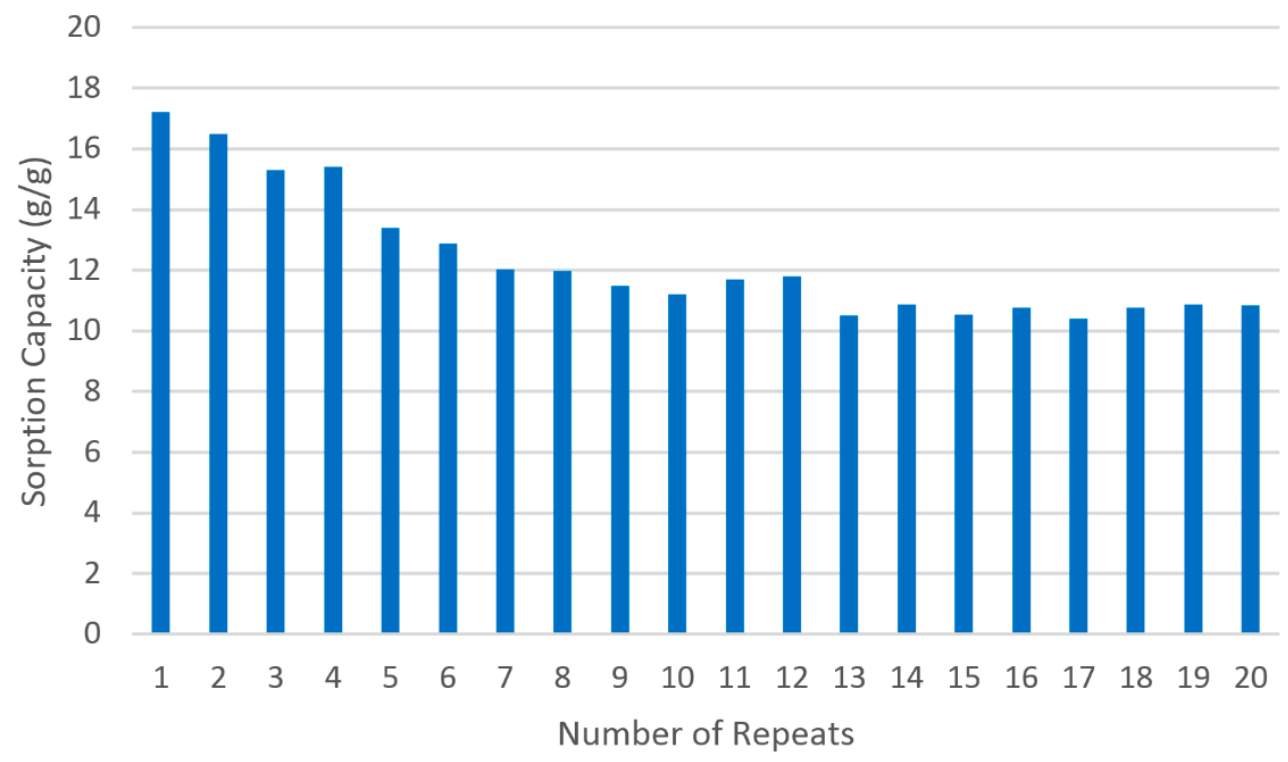

Figure 9. Recyclability test for functionalised cigarette filters using motor oil.

The recyclability results showed very good recyclability when compared with the work of Jin et al. [24], where the adsorption capacity of cellulose-based aerogels from waste newspaper lost over $93 \%$ of their adsorption capacity after five recyclability tests using squeezing, which implied a total collapse of the porous structures. The filters, however, compared favorably with the work of $\mathrm{Ou}$ et al. [35], who achieved up to $96 \%$ capacity after 10 cycles of reused of hexadecyltrimethoxysilane-modified cigarette filters for the removal of kerosene, and Han et al. [23], 
who recorded a reduction of $31.5 \%$ in the adsorption capacity of carbon aerogels from waste newspaper using the squeezing method, a reduction of $3 \%$ using the "combustion in air" method, and an adsorption capacity that stayed unaltered using the distillation method, all after five recyclability cycles. Huang et al. [28] recorded a 30\% loss of adsorption capacity from multi-walled carbon nanotube-silica aerogels in their study after 15 adsorption capacity cycles. Zhu et al. [36] fabricated porous carbon aerogels from pomelo peels, which showed a reduction of $28 \%$ after five recyclability cycles using the solvent extraction method. The results of this work show that waste cigarette filters have excellent recyclability when compared to fabricated materials from other studies, as the sorbents continued to adsorb oil even after 20 recyclability cycles, making them an excellent low-cost alternative to the more advanced sorbent materials.

\section{Conclusions}

Waste cigarette filters are an environmental menace. In this research, they were used as raw material for the simple fabrication of a superhydrophobic and oleophilic sorbent for application in oil/water separation and spill clean-up. Following the cleaning and hydrolysis of the filters, they were surface-functionalised by MTCS, using vapor phase deposition to improve the hydrophobicity of the adsorbent. The functionalisation process transformed the adsorbent into a superhydrophobic material with a water contact angle of $154 \pm 3.5^{\circ}$. The fabricated filters achieved an oil sorption capacity of 25.6, 15.8, 17.2, and $21.5 \mathrm{~g} / \mathrm{g}$ for vegetable oil, diesel oil, motor oil, and VP oil, respectively. The sorbent oil sorption capacity did not significantly deteriorate after 20 cycles of reuse, indicating the potential of the waste filters as a high sorption and recyclable sorbent for oil spill clean-up while providing a means of eliminating an environmental pollutant.

Author Contributions: A.O.I. conceived and designed the experiments, A.O.I. and E.E.L. carried out the laboratory experiments, and F.U.M. and S.C.T. assisted in putting the paper together.

Funding: This research received no external funding.

Acknowledgments: The authors are grateful to the School of Energy Construction and Environment for material support.

Conflicts of Interest: The authors declare no conflict of interest.

\section{References}

1. Ifelebuegu, A.O.; Ukpebor, J.E.; Ahukannah, A.U.; Nnadi, E.O.; Theophilus, S.C. Environmental effects of crude oil spill on the physicochemical and hydrobiological characteristics of the Nun River, Niger Delta. Environ. Monit. Assess. 2017, 189, 173. [CrossRef] [PubMed]

2. Somasundaran, P.; Patra, P.; Farinato, R.S.; Papadopoulos, K. Oil Spill Remediation: Colloid Chemistry-Based Principles and Solutions; John Wiley \& Sons: Hoboken, NJ, USA, 2014.

3. Ifelebuegu, A.O.; Johnson, A. Non-conventional low-cost cellulose and keratin based biopolymeric sorbents for oil/water separation and spill clean-up: A Review. Crit. Rev. Environ. Sci. Technol. 2017, 47, 964-1001. [CrossRef]

4. Feng, J.; Nguyen, S.T.; Fan, Z.; Duong, H.M. Advanced Fabrication and Oil Absorption Properties of Super-Hydrophobic Recycled Celluose Aerogels. Biochem. Eng. J. 2015, 270, 168-175.

5. Speight, J.G.; Arjoon, K.K. Bioremediation of Petroleum and Petroleum Products; John Wiley \& Sons: Hoboken, NJ, USA, 2012.

6. Zhao, J.; Xiao, C.; Feng, Y.; Xu, N. A Review: Polymethacrylate Fibres as Oil Absorbents. Polym. Rev. 2013, 53, 527-545. [CrossRef]

7. Wan, C.; Lu, Y.; Jiao, Y.; Jin, C.; Sun, Q.; Li, J. Fabrication of Hydrophobic Electrically Conductive and Flame-Resistant Carbon Aerogels by Pyrolysis of Regenerated Cellulose Aerogels. Carbohydr. Polym. 2015, 118, 115-118. [CrossRef] [PubMed]

8. Ifelebuegu, A.O.; Tuan Vu Anh Nguyen, P.U.I.; Zenebu, M. Liquid-phase sorption characteristics of human hair as a natural oil spill sorbent. J. Environ. Chem. Eng. 2015, 3, 938-943. [CrossRef] 
9. Sabir, S. Approach of Cost Effective Absorbents for Oil Removal from Oily Water. Crit. Rev. Environ. Sci. Technol. 2015, 45, 1916-1945. [CrossRef]

10. Sarbatly, R.; Krishnaiah, D.; Kamin, Z. A Review of polymer nano-fibres by electrospinning and their application in oil-water separation for cleaning up marine oil spills. Mar. Pollut. Bull. 2016, 106, 8-16. [CrossRef] [PubMed]

11. Kushwaha, S.; Soni, H.; Ageetha, V.; Padmaja, P. An insight into the production, characterization, and mechanisms of action of low-cost adsorbents for removal of organics from aqueous solution. Crit. Rev. Environ. Sci. Technol. 2013, 43, 443-549. [CrossRef]

12. Novotny, T.E.; Lum, K.; Smith, E.; Wang, V.; Barnes, R. Cigarette Butts and the Case for an Environmental Policy on Hazardous Cigarette Waste. Int. J. Environ. Res. Public Health 2009, 6, 1691-1705. [CrossRef] [PubMed]

13. Gould, H. Why Cigarette Butts Threaten to Stub Out Marine Life. Available online: https: / /www.theguardian.com/sustainable-business/2015/jun/09/why-cigarette-butts-threaten-to-stub-outmarine-life (accessed on 16 June 2017).

14. Lee, J. Get Your Butt Off the Ground: Consequences of Cigarette Waste and Litter-Reducing Methods. Available online: http:/ / scholarship.claremont.edu/cgi/viewcontent.cgi?article=1043\&context=pomona_ theses (accessed on 16 June 2018).

15. Slaughter, E.; Gersberg, R.M.; Watanabe, K.; Rudolph, J.; Stransky, C.; Novotny, T.E. Toxicity of Cigarette Butts and their Chemical Components to Marine and Freshwater Fish. Tob. Control 2011, 20, 25-29. [CrossRef] [PubMed]

16. Kabasci, S. Bio-Based Plastics-Materials and Applications; John Wiley \& Sons: Hoboken, NJ, USA, 2014.

17. Zhao, J.; Zhang, N.S.; Qu, C.T.; Wu, X.M.; Zhang, J.T.; Zhang, X. Cigarette butts and their application in corrosion inhibition for $\mathrm{N} 80$ steel at $90{ }^{\circ} \mathrm{C}$ in a hydrochloric acid solution. Ind. Eng. Chem. Res. 2010, 49, 3986-3991. [CrossRef]

18. Liu, C.; Chen, B.; Yang, J.; Li, C. One-step fabrication of superhydrophobic and superoleophilic cigarette filters for oil-water separation. J. Adhes. Sci. Technol. 2015, 29, 2399-2407. [CrossRef]

19. He, F.; Sui, C.; He, X.; Li, M. Facile Synthesis of Strong Alumina-Cellulose Aerogels by Freeze-Drying Method. Mater. Lett. 2015, 152, 9-12. [CrossRef]

20. Lim, M.B.; Hu, M.; Manandhar, S.; Sakshaug, A.; Strong, A.; Riley, L.; Pauzauskie, P.J. Ultrafast Sol-Gel Synthesis of Graphene Aerogel Materials. Carbon 2015, 95, 616-624. [CrossRef]

21. Cao, N.; Lyu, Q.; Li, J.; Wang, Y.; Yang, B.; Szunerits, S.; Boukherroub, R. Facile Synthesis of Flourinated Polydopamine/Chitosan/Reduced Graphene-Oxide Composite Aerogel for Efficient Oil/Water Separation. Chem. Eng. J. 2017, 326, 17-28. [CrossRef]

22. Ren, R.P.; Li, W.; Lv, Y.K. A Robust, Super Hydrophobic Graphene Aerogel as a Recyclable Sorbent for Oils and Organic Solvents at Various Temperatures. J. Colloid Interface Sci. 2017, 500, 63-68. [CrossRef] [PubMed]

23. Han, S.; Sun, Q.; Zheng, H.; Li, J.; Jin, C. Green and Facile Fabrication of Carbon Aerogels from Cellulose-Based Waste Newspaper for Solving Organic Pollution. Carbohydr. Polym. 2016, 136, 95-100. [CrossRef] [PubMed]

24. Jin, C.; Han, S.; Li, J.; Sun, Q. Fabrication of Cellulose-Based Aerogels from Waste Newspaper without any Pre-treatment and their use for Absorbents. Carbohydr. Polym. 2015, 123, 150-156. [CrossRef] [PubMed]

25. Nguyen, S.T.; Feng, J.; Ng, S.K.; Wong, J.P.W.; Tan, V.B.C.; Duong, H.M. Advanced Thermal Insulation and Absorption Properties of Recycled Cellulose Aerogels. Colloids Surf. A 2014, 445, 128-134. [CrossRef]

26. Schmitt, M. Analysis of silanes and of siloxanes formation by Raman spectroscopy. RSC Adv. 2014, 4, 1907-1917. [CrossRef]

27. Munief, W.M.; Heib, F.; Hempel, F.; Lu, X.; Schwartz, M.; Pachauri, V.; Hempelmann, R.; Schmitt, M.; Ingebrandt, S. Silane deposition via gas-phase evaporation and high-resolution surface characterization of the ultra-thin siloxane coatings. Langmuir 2018. [CrossRef] [PubMed]

28. Huang, J.; Liu, H.; Chen, S.; Ding, S. Hierarchical Porous MWCNTs-Silica Aerogel Synthesis for High-Efficiency Oily Water Treatment. J. Environ. Chem. Eng. 2016, 4, 3274-3282. [CrossRef]

29. Wei, Q.F.; Mather, R.R.; Fotheringham, A.F.; Yang, R.D. Evaluation of nonwoven polypropylene oil sorbents in marine oil-spill recovery. Mar. Pollut. Bull. 2003, 46, 780-783. [CrossRef] 
30. Wang, J.; Geng, G.; Wang, A.; Liu, X.; Du, J.; Zou, Z.; Zhang, S.; Han, F. Double biomimetic fabrication of robustly superhydrophobic cotton fiber and its application in oil spill cleanup. Ind. Crops Prod. 2015, 77, 36-43. [CrossRef]

31. Ifelebuegu, A.O.; Momoh, Z. An Evaluation of the Adsorptive Properties of Coconut Husk for Oil Spill Cleanup International. J. Chem. Eng. 2015, 2, 115-119.

32. Liao, Q.; Su, X.; Zhu, W.; Hua, W.; Qian, Z.; Liu, L.; Yao, J. Flexible and durable cellulose aerogels for highly effective oil/water separation. RSC Adv. 2016, 6, 63773-63781. [CrossRef]

33. Angelova, D.; Uzunov, I.; Uzunova, S.; Gigova, A.; Minchev, L. Kinetics of oil and oil products adsorption by carbonized rice husks. Chem. Eng. J. 2011, 172, 306-311. [CrossRef]

34. Xiao, N.; Zhou, Y.; Ling, Z.; Qiu, J. Synthesis of a carbon nanofiber/carbon foam composite from coal liquefaction residue for the separation of oil and water. Carbon 2013, 59, 530-536. [CrossRef]

35. Ou, J.; Wan, B.; Wang, F.; Xue, M.; Wu, H.; Li, W. Superhydrophobic fibers from cigarette filters for oil spill cleanup. RSC Adv. 2016, 50, 44469-44474. [CrossRef]

36. Zhu, L.; Wang, Y.; Wang, Y.; You, L.; Shen, X.; Li, S. An Environmentally Friendly Carbon Aerogel Derived from Waste Pomelo Peel for the Removal of Organic Pollutants/Oils. Microporous Mesoporous Mater. 2017, 241, 285-292. [CrossRef]

(C) 2018 by the authors. Licensee MDPI, Basel, Switzerland. This article is an open access article distributed under the terms and conditions of the Creative Commons Attribution (CC BY) license (http:/ / creativecommons.org/licenses/by/4.0/). 